\title{
Editorial
}

\section{Resposta imediata da Vigilância em Saúde à epidemia da COVID-19}

doi: 10.5123/S1679-49742020000100021

\author{
Immediate Health Surveillance Response to COVID-19 Epidemic \\ Respuesta inmediata de la Vigilancia en Salud a la epidemia de COVID-19
}

$\mathrm{E}$ m 31 de dezembro 2019, foi notificada à Organização Mundial da Saúde (OMS) a ocorrência de um surto de pneumonia na cidade de Wuhan, província de Hubei, República Popular da China. Rapidamente, identificou-se o agente etiológico, um novo coronavírus: SARS-COV-2 0 surto teve início em um mercado de frutos do mar e animais vivos e, até o momento desta publicação, o reservatório animal é desconhecido. ${ }^{1}$

A primeira reunião do Comitê de Emergência sobre o surto do novo coronavírus na China, convocada pela OMS de acordo com o Regulamento Sanitário Internacional (RSI) (2005), ocorreu em 23 de janeiro de 2020. Nessa reunião, não houve consenso sobre se o evento constituía uma Emergência de Saúde Pública de Importância Internacional (ESPII). ${ }^{2} \mathrm{Na}$ segunda reunião, realizada em 30 de janeiro, foi constatado o crescimento no número de casos e de países que reportaram casos confirmados, o que levou à declaração do surto como ESPII. ${ }^{3}$

Em fevereiro de 2020, de acordo com as melhores práticas da OMS para nomear novas doenças infecciosas humanas, a doença causada pelo novo coronavírus recebeu a denominação COVID-19, em referência ao tipo de vírus e ao ano de início da epidemia: Coronavirus disease - 2019. ${ }^{4}$ Até o final desse mês, eram quase 80 mil casos confirmados e 2.838 óbitos por COVID-19 na China e, mais aproximadamente, 6 mil casos confirmados e 86 óbitos em outros 53 países.

No Brasil, o primeiro caso de COVID-19 foi confirmado em 26 de fevereiro de 2020. Em 3 de março, havia 488 casos suspeitos notificados, 2 confirmados e 240 descartados no país, sem evidência de transmissão local. Os dois primeiros casos confirmados eram de indivíduos do sexo masculino, residentes na cidade de São Paulo, SP, que haviam regressado de viagem à Itália.

A resposta da Secretaria de Vigilância em Saúde do Ministério da Saúde (SVS/MS) à epidemia da COVID-19 foi imediata. No mesmo dia 3 de janeiro, a partir da detecção de rumores, foram acionados os Pontos Focais Nacionais do Regulamento Sanitário Internacional da OMS (PFN-RSI/OMS). Após avaliação de risco, o evento foi incluído pelo Comitê de Monitoramento de Eventos em 10 de janeiro. Em 22 de janeiro, foi acionado o Centro de Operações de Emergência (COE) do Ministério da Saúde, coordenado pela SVS/MS, para harmonização, planejamento e organização das atividades com os atores envolvidos e o monitoramento internacional. Em 27 de janeiro, foi ativado o plano de contingência e, em 3 de fevereiro a epidemia foi declarada Emergência em Saúde Pública de Importância Nacional (ESPIN). ${ }^{5}$

Ainda no mês de janeiro, foram publicados pela SVS/MS três Boletins Epidemiológicos sobre o tema, com foco nas ações de prevenção e enfrentamento. Esses Boletins trazem, outrossim, as definições atualizadas de caso suspeito, segundo critérios clínicos e epidemiológicos. A Plataforma IVIS disponibiliza informações diariamente, sobre o número atual de casos suspeitos, descartados e confirmados, em seu sítio eletrônico (http://plataforma.saude.gov.br/novocoronavirus/). Ademais, foram frequentes as entrevistas e comunicados à imprensa e à sociedade, destacando-se o cuidado da SVS/MS com a transparência na informação e agilidade na comunicação sobre 0 evento.

0 governo federal também reagiu prontamente. Em 30 de janeiro, foi publicado o Decreto $\mathrm{n}^{0} 10.211$, que reativa o Grupo Executivo Interministerial de Emergência em Saúde Pública de Importância Nacional e Internacional (GEI-ESPII). ${ }^{6}$ Entre as atribuições do GEI-ESPII, está a articulação de medidas de preparação e de 
enfrentamento às emergências em Saúde Pública no âmbito nacional e internacional. 0 GEI-ESPII, coordenado pelo Ministério da Saúde, é composto por representantes dos seguintes órgãos e entidades: Casa Civil; Ministério da Justiça e Segurança Pública; Ministério da Defesa; Ministério da Agricultura, Pecuária e Abastecimento; Ministério do Desenvolvimento; Gabinete de Segurança Institucional; e Agência Nacional de Vigilância Sanitária (Anvisa). No mesmo dia 30 de janeiro, o Ministério da Saúde anunciou a abertura de processo de licitação para alocar mil leitos adicionais nos hospitais de referência indicados pelos estados, para atender a possíveis casos de COVID-19.

Também foi anunciada a publicação de edital de processo para aquisição de equipamentos de proteção individual (EPI) destinados aos profissionais de saúde - como máscaras cirúrgicas, protetores faciais, gorros, máscaras N95 e luvas -, além de outros insumos. A proteção à saúde dos trabalhadores é fundamental, uma vez reconhecida a propensão para propagação de coronavírus nos serviços de saúde. ${ }^{7}$

De forma semelhante aos surtos causados por dois outros coronavírus respiratórios humanos patogênicos - coronavírus da síndrome respiratória aguda grave (SARS-CoV) e coronavírus da síndrome respiratória do Oriente Médio (MERS-CoV) -, o SARS-COV-2 é transmitido de humano para humano e capaz de causar doença respiratória grave; contudo, ele se distingue pela capacidade de transmissão a partir de casos assintomáticos, fato que, somado à existência de uma proporção todavia desconhecida de doentes que não desenvolvem manifestações graves da doença, são fatores que afetam a capacidade de contenção da propagação do vírus. ${ }^{7}$

Estudo de modelagem apontou a possibilidade, na ausência de intervenções de Saúde Pública em larga escala, da ocorrência de surtos independentes e autossustentáveis da COVID-19 nas principais cidades do mundo, haja vista a exportação substancial de casos pré-sintomáticos. ${ }^{8}$ No cenário descrito, ações de resposta como estas, em desenvolvimento pela Vigilância em Saúde do Brasil, são fundamentais para conter a propagação do vírus e da doença.

\footnotetext{
Julio Henrique Rosa Croda ${ }^{1}$ - D orcid.org/0000-0002-6665-6825

Leila Posenato Garcia² - (1) orcid.org/0000-0003-1146-2641
}

'Ministério da Saúde, Secretaria de Vigilância em Saúde, Departamento de Imunização e Doenças Transmissíveis, Brasília, DF, Brasil

${ }^{2}$ Ministério da Economia, Instituto de Pesquisa Econômica Aplicada, Diretoria de Estudos e Políticas Sociais, Brasília, DF, Brasil

\section{Referências}

1. Zhu N, Zhang D, Wang W, Li X, Yang B, Song J, et al. A novel coronavirus from patients with pneumonia in China, 2019. N Engl J Med [Internet]. 2020 Feb [cited 2020 Mar 4];382:727-33. Available from: http://doi.org/10.1056/ NEJMoa2001017

2. World Health Organization. Statement on the meeting of the International Health Regulations (2005) Emergency Committee regarding the outbreak of novel coronavirus (2019-nCoV) [Internet]. Geneva: World Health Organization; 2020 [cited $2020 \mathrm{Mar} 4$ ] . Availabe from: https://www.who.int/news-room/detail/23-01-2020-statement-on-themeeting-of-the-international-health-regulations-(2005)-emergency-committee-regarding-the-outbreak-of-novelcoronavirus-(2019-ncov)

3. World Health Organization. Statement on the second meeting of the International Health Regulations (2005) Emergency Committee regarding the outbreak of novel coronavirus (2019-nCoV) [Internet]. Geneva: World Health Organization; 2020 [cited 2020 Mar 4] . Available from: https://www.who.int/news-room/detail/30-01-2020-statementon-the-second-meeting-of-the-international-health-regulations-(2005)-emergency-committee-regarding-the-outbreakof-novel-coronavirus-(2019-ncov)

4. World Health Organization. Novel coronavirus (2019-nCoV): situation report - 22 [Internet]. Geneva: World Health Organization; 2020 [cited 2020 Mar 4]. Available from: https:/www.who.int/docs/default-source/coronaviruse/ situation-reports/20200211-sitrep-22-ncov.pdf?sfvrsn=fb6d49b1_2 
5. Brasil. Ministério da Saúde. Portaria MS/GM no 188 , de 3 de fevereiro de 2020. Declara Emergência em Saúde Pública de importância Nacional (ESPIN) em decorrência da Infecção Humana pelo novo Coronavírus (2019-nCoV) [Internet]. Diário Oficial da União, Brasília (DF), 2020 fev 4 [citado 2020 mar 4]; Seção Extra:1. Disponível em: http://www.in.gov.br/web/dou/-/portaria-n-188-de-3-de-fevereiro-de-2020-241408388

6. Brasil. Presidência da República. Casa Civil Decreto no 10.211, de 30 de janeiro de 2020. Dispõe sobre o Grupo Executivo Interministerial de Emergência em Saúde Pública de Importância Nacional e Internacional - GEI-ESPII [Internet]. Diário Oficial da União, Brasília (DF), 2020 jan 30 [citado 2020 mar 4];Seção 1- Extra;1. Disponível em: http://www.in.gov.br/web/dou/-/decreto-n-10.211-de-30-de-janeiro-de-2020-240646239?inheritRedirect=true\&redirec $\mathrm{t}=\% 2$ Fweb\%2Fguest\%2Fsearch\%3Fseca0\%3Ddou1\%26data\%3D31-01-2020\%26qSearch\%3DGei

7. Munster VJ, Koopmans M, van Doremalen N, van Riel D, Wit E. A novel coronavirus emerging in china - key questions for impact assessment. N Eng J Med [Internet]. 2020 Feb [cited 2020 Mar 4];382:692-4. Available from: https://www. nejm.org/doi/full/10.1056/NEJMp2000929?query=TOC

8. Wu JT, Leung K, Leung GM. Nowcasting and forecasting the potential domestic and international spread of the 2019-nCoV outbreak originating in Wuhan, China: a modelling study. Lancet [Internet]. 2020 Jan [cited 2020 Mar 4];395(10225):689-97. Available from: https://doi.org/10.1016/S0140-6736(20)30260-9 